\title{
Simulating bio-composite cycling helmet performance through FEA and CFD approaches
}

\begin{abstract}
Finite Element Analysis (FEA) and Computational Fluid Dynamics (CFD) analysis were performed in this work in order to obtain the best design for safety and aerodynamic performance of the bicycle cycling helmet. FEA analysis was computed on two different helmet designs to determine the critical area subjected to impact. A pressure load was applied on the helmetsô outer surface to simulate oblique loading. The critical areas of the helmets were then highlighted and identified, enabling design improvements to be made on both designs. CFD analysis was then executed in order to obtain the lowest drag coefficient number in reducing the air resistance induced by both of the helmet designs, inherently increasing cyclist performance and ensuring competition success.
\end{abstract}

Keyword: Bio-composite cycling helmet; Cycling helmet performance; Finite Element Analysis (FEA); Computational Fluid Dynamics (CFD) 